\title{
Ludolph Brauer - ein moderner Internist und Pneumologe
}

\author{
Ludolph Brauer - a Leader in Modern Internal Medicine and Chest Medicine
}

Autor

Institut

\section{P. v. Wichert}

ehem. Med. Poliklinik der Philipps-Universität Marburg

\section{Bibliografie}

Dol $10.1055 / \mathrm{s}-0028-1119700$ Online-Publikation: 16. 3. 2009 Pneumologie 2009; 63: 492-496 @ Georg Thieme Verlag KG Stuttgart · New York ISSN 0934-8387

Korrespondenzadresse Prof. Dr. Peter v. Wichert Eppendorfer Landstr. 14 20249 Hamburg

vonwichert@t-online.de
Gedanken über die Entwicklung eines wissenschaftlichen Gebietes sind immer interessant und lehrreich. Man erkennt die Entwicklung der Medizin, man kann rückblickend sehen, welche Fehler hätten vermieden werden können und wo die Chancen waren, aber auch welche Überlegungen schließlich zum Fortschritt beigetragen haben. Sehr häufig lassen sich die Entwicklungen anhand der sie tragenden Persönlichkeiten erkennen. Das gilt auch für die Wissenschaft von den Erkrankungen der Lunge. Nicht zuletzt sind historische Kenntnisse aber auch deswegen wertvoll, weil sie zeigen, dass nicht alles, was wir heute tun, aus dem Ausland kommt, sondern dass vieles, was heute Standard in der Medizin ist, in Deutschland vorgedacht und gemacht oder entwickelt wurde. Diese Überlegungen treffen besonders auf das wissenschaftliche Werk von Ludolph Brauer ( $\bullet$ Abb. 1) zu.

Dessen Bedeutung für die Medizin wieder mehr in den Fokus des Wissens um die Entwicklung unseres Faches und der Gegenwartsmedizin zu rücken, ist Anliegen der nachfolgenden Ausführungen.

Ludolph Brauer ist ohne jeden Zweifel einer der bedeutendsten Internisten der ersten Hälfte des 20. Jahrhunderts, und das nicht nur auf Deutschland bezogen, sondern weltweit. Er wurde am 1.7.1865 in Hohenhausen bei Thorn (Westpreußen) als Sohn eines Gutsbesitzers geboren. Seine Jugend verbrachte er in Bremen. Möglicherweise hat die Kombination aus preußischer Herkunft und hanseatischer Bildung zu seiner Charakterstruktur beigetragen. Nach einem Studium in Freiburg war er Assistent in Bonn und Heidelberg und habilitierte sich dort unter Erb 1897. 1901 wurde er außerordentlicher Professor in Heidelberg. In Heidelberg hatte er über Leber- und Pankreasfunktionen, über Kreislauffunktionen und Herzerkrankungen und über den Respirationstrakt gearbeitet. Er war ein sehr vielseitig interessierter Wissenschaftler und Arzt, in einer Weise

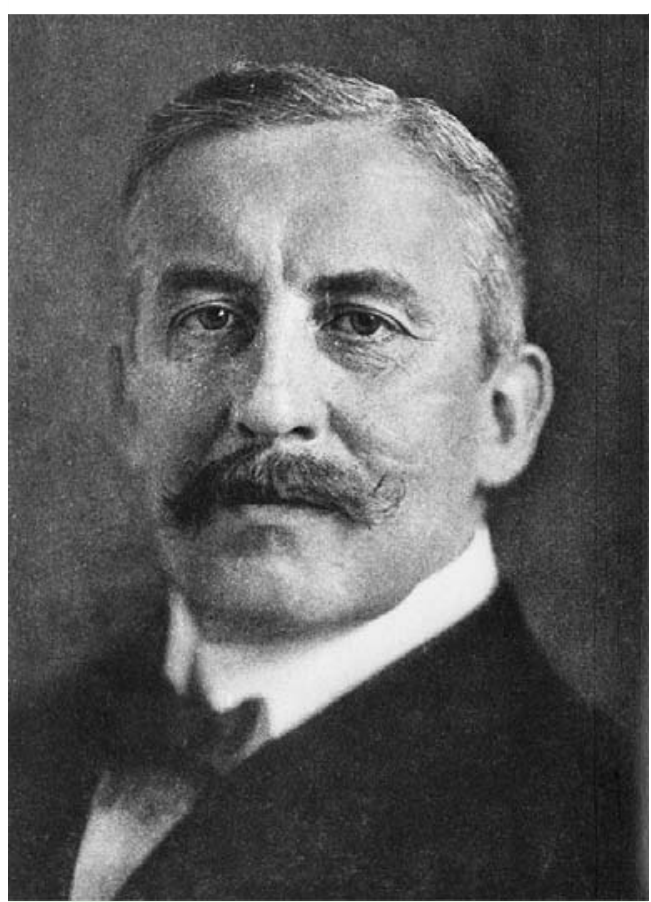

Abb. 1 Ludolph Brauer.

vielseitig, wie man es heute kaum noch irgendwo findet, in der ganzen Breite der Inneren Medizin tätig und auch die ganze Breite der Inneren Medizin mit Ideen anreichernd.

Damals war eines der größten Gesundheitsprobleme die Therapie der Tuberkulose. Nach der Entdeckung des Bakteriums durch Robert Koch (1882) war die Krankheit ätiologisch aufgeklärt, aber therapeutisch nur marginal zu beherrschen. Nur durch operative Entfernung der befallenen Areale der Lunge hoffte man dieser Erkrankung Herr werden zu können, was zu einer intensiven Beschäftigung mit der Thoraxchirurgie führte. Dagegen standen aber die Gefahren, die sich durch die Eröffnung des Pleuraraumes ergaben. Eine Eröffnung des Thorax, eine Voraussetzung für einen operativen Eingriff an der Lunge, wurde 
(Aus der kgl. chirurgischen Klinik und dem pharmazeutischen Institut zu Breslau, Prof. v. Mikulicz und Prof. Filehne.)

Über die Ausschaltung der schädlichen Wirkung des Pneumothorax bei intrathorakalen Operationen. Von

\section{Dr. Ferdinand Sauerbruch,} wissensehaftlicher Assistent der Klinik.

Im Oktober vorigen Jahres gab mir Herr Geh.-Rat v. Mikulicz die Anregung zu einer experimentellen Arbeit über die Möglichkeit, bei intrathorakischen Operationen die schädliche Wirkung des Pneumothorax auszuschalten. Er hatte dabei in erster Linie die transpleurale Ösophagusresektion im Auge, welche bisher sowohl am Menschen als auch im Tierexperiment die traurigsten Resultate ergeben hat. Eine der Hauptursachen der Mißerfolge war der mit der Operation verbundene Pneumothorax und die funktionelle Ausschaltung der unter dem atmosphärischen Druck kollabierten Lunge. Da bei Hunden die Resektion des unteren Ösophagusabschnittes fast immer zur Eröffnung der zweiten Pleurahöhle führt, so verendet ein großer Teil der so operierten Tiere noch auf dem Operationstische.

Abb. 2

Aus der medizinischen Klinik zu Heidelberg (Direktor: Geh.-Rat ERB).

Nachdruck verboten

\section{XVIII.}

\section{Die Ausschaltung der Pneumothoraxfolgen mit Hilfe des Ueberdruckverfahrens ${ }^{1}$ ). Von \\ Dr. L. Brauer, \\ a. 0. Professor in Heidelberg. \\ (Hierzu 2 Abbildungen im Texte.)}

Im Centralblatt für Chirurgie, 1904, Heft 6, gab SaUerbruch ein Verfahren zur Ausschaltung der schädlichen Wirkungen des Pneumothorax bei intrathorakalen Operationen bekannt. Der Autor bemühte sich, da die bisher üblichen Methoden der künstlichen Atmung sich als unzulänglich erwiesen hatten, die schädlichen Folgen des atmosphärischen Druckes auf der Lungenaußenfläche dadurch auszuschalten, daß er einen entsprechenden negativen Druck in demjenigen Teile des Operationsraumes herstellte, mit welchem die zu eröffnende Pleurahöhle in offene Verbindung zu treten hat. Zunächst konstruierte SAUERBRUCH, wie er berichtet, einen primitiven Apparat, welcher diesen Anforde-

Abb. 3

sehr gefürchtet, denn der Kollaps der Lunge nach Öffnung des Thorax verminderte einerseits die Möglichkeiten des Operateurs, aber andererseits auch die Atemfläche und den venösen Rückstrom zum Herzen mit Folgen für die Kreislaufstabilität. Das wissenschaftliche Problem war international virulent. Es wurde an zwei Stellen einer Lösung zugeführt, in Breslau, später Marburg, Zürich und Berlin durch Ferdinand Sauerbruch und in Heidelberg, später Marburg und Hamburg durch Ludolph Brauer. Sauerbruch hat dieses Problem durch die Unterdruckkonstruktion seiner OP-Kammer zum Teil gelöst, während Brauer die Anwendung von Überdruck per Kopfmaske propagierte. Wie wir wissen, hat sich die Methode der Überdruckbeatmung weltweit durchgesetzt. Seinerzeit war die nicht unberechtigte Sorge die, dass bei einer Überdruckanwendung der venöse Rückstrom zum Herzen mit sehr nachteiligen Konsequenten für den Gesamtorganismus gestört sein könnte. Wir wissen heute, dass das auch der Fall ist, sich aber nicht gefährlich auswirkt. Ludolph Brauer hat in einer Vielzahl von Experimenten gezeigt, dass sich seine Technik
1) In meinem Aufsatze „Ueber Operationen in der Brusthöhle mit Hilfe der Sauerbruchschen Kammer" in No, 15 u. 16 der Dtsch. med. Wochenschrift 1904 habe ich der Prioritätsfrage Erwähnung getan, welche zwischen verfahrens, d. h. der Umkehrung des ursprünglich SAUERBRUchschen Verfahrens, entstanden ist. Ich benutze gern diese Gelegenheit, um ausdrücklich hervorzuheben, daß durch die uns gegebenen Aufklärungen einwandsfrei dargelegt ist, daß Herr Prof. Brauer ganz unabhängig und ohne von unseren dahingehenden Versuchen Kenntnis zu haben, auf die Idee des Ueberdruckverfahrens gekommen ist und dieselbe technisch durchzuführen unternommen hat. Es ist kein Zweifel, daß, wenn sich das Ueberdruckverfahren nicht nur im Tierexperiment, sondern auch für den Menschen bewăhren sollte, dies eine wesentliche technische Vereinfachung der ganzen Methode bedeuten würde. v. Mikulicz.

Abb. 4

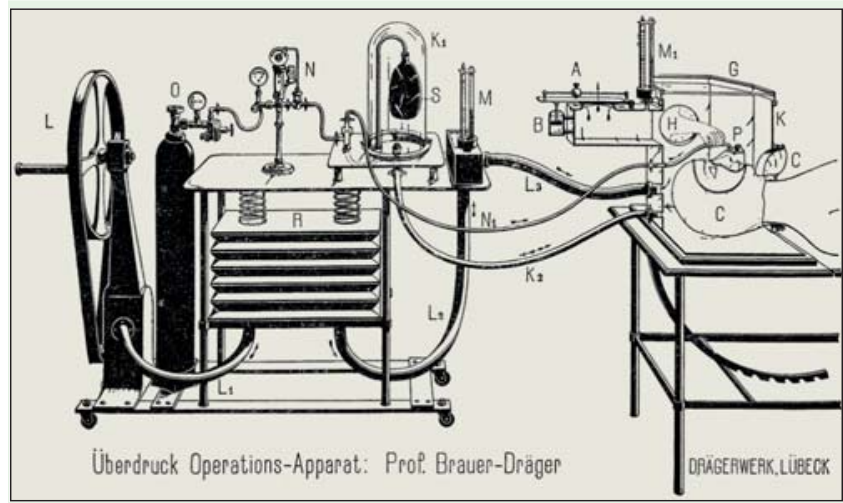

Abb. 5

durchaus auch zur Anwendung am Patienten eignet. Dass er sich zunächst gegen Sauerbruch nicht durchsetzen konnte, ist ein Beispiel für die Bedeutung eines wissenschaftlichen „main streams“. Wie nah sich diese gegensätzlichen Methoden aber andererseits auch waren, kann man in einer Ausgabe der Zeitschrift für „Grenzgebiete der Inneren Medizin und Chirurgie“ nachlesen. 1904 haben Brauer und Sauerbruch, die Arbeiten folgen einander, ihre Methoden publiziert ( $\bullet$ Abb. 2, 3).

Interessant ist das Nachwort des Herausgebers, F. v. Mikulicz, Direktor der Chirurgie in Breslau und Chef von F. Sauerbruch: dass, wenn sich die Methode von Brauer als effektiv herausstellen würde, sie die geeignetere Wahl bei einer Operation am offenen Thorax wäre $(\bullet$ Abb. 4).

Es ist eine eigenartige Fügung der Geschichte, dass sich die Überdruckbeatmung bei Narkose erst nach dem 2. Weltkrieg über die USA wieder flächendeckend in Deutschland etablierte - aber jetzt unter endotrachealer Intubation, nicht unter Spontanatmung -, obwohl sie hier durch Brauer inauguriert worden war. Dieses Verfahren entwickelt und mit der ihm eigenen Energie auch gegen Widerstände zur Praxisreife gebracht zu haben, veranschaulicht besonders eindrucksvoll die wissenschaftlich drängende Kraft, die Ludolph Brauer auszeichnete, andererseits aber auch die hohe wissenschaftliche Qualität der akademischen Medizin zu Beginn des 20. Jahrhunderts in Deutschland.

Der in der $\bullet$ Abb. 5 gezeigte Beatmungsapparat der Firma Dräger in Lübeck war funktionsfähig, allerdings war die Intubation noch nicht zum Standard geworden.

Nach seiner Berufung nach Marburg hat Brauer dieses Verfahren in der DMW 1905 veröffentlicht [4], die erste Publikation der heute international als Routine durchgeführten Überdruckbeatmung ( $\bullet$ Abb. 6).

Ludolph Brauer wurde am 1.10.1904 zum außerordentlichen Professor für Innere Medizin und zum Direktor der Medizinischen Poliklinik der Universität Marburg als Nachfolger von Ernst 


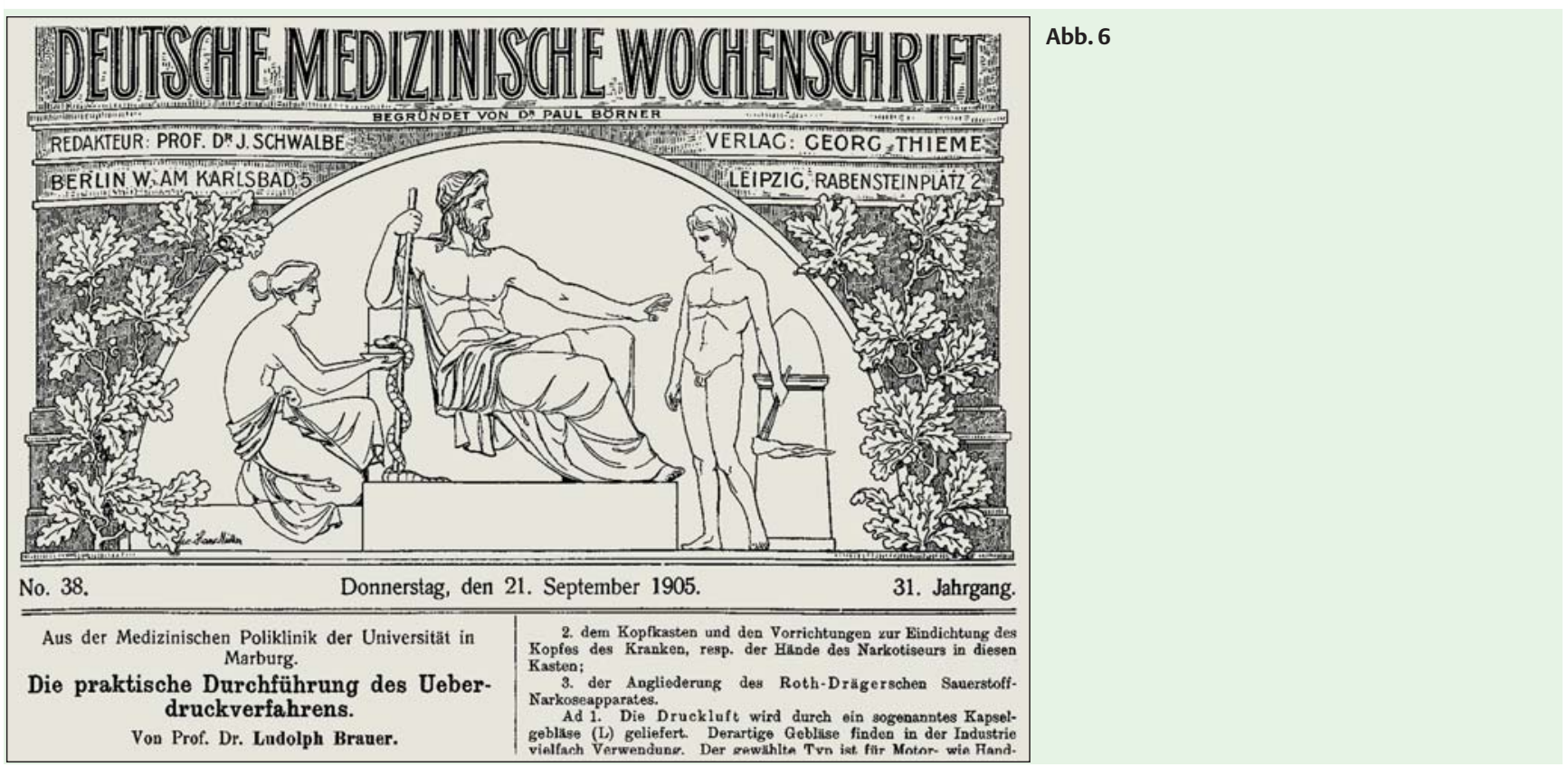

Romberg berufen und am 1.8.1905 in der Nachfolge von Emil Mannkopff gleichzeitig zum Direktor auch der Medizinischen Klinik ernannt (eine Position, die der Autor dieser Zeilen in zeitentsprechender Weise 75 Jahre später ebenfalls innehatte).

Ludolph Brauer hatte schon in Heidelberg und später in Marburg seine Auffassung von einer wissenschaftlichen Medizin und wesentliche Grundzüge seiner Überlegungen zu den Strukturen der klinischen Medizin deutlich gemacht, wie seine Antrittsvorlesung als Direktor der Medizinischen Klinik in Marburg, im Oktober 1905 zeigt. Er beschreibt in dieser, in der DMW abgedruckten Rede [5] die auch heute noch im Prinzip gültigen Anforderungen, die an einen Direktor einer Klinik gestellt werden müssen und die auch heute noch nicht völlig geregelten und verstandenen Beziehungen zwischen dem Gesamtfach Innere Medizin und den einzelnen Schwerpunkten des Faches. Er bejaht eine Spezialisierung, erwartet aber, dass das Interesse und die Kenntnis im Gesamtfach so gepflegt werden, dass sich die Ärzte in den Subspezialitäten untereinander verstehen und alle an der Mehrung des Faches Innere Medizin aktiv beteiligt sind. Ludolph Brauer hat die Innere Medizin als Gesamtfach nie in Frage gestellt, obwohl er wie kaum ein anderer auf einem sehr spezialisierten Gebiet seine besonderen Erfolge hatte, als einer der international herausragenden Forscher auf dem Gebiet der Lungenkrankheiten, ausgezeichnet mit einer Fülle internationaler Ehrungen.

Ludolph Brauer hat für die Innere Medizin und die Pneumologie, die damals noch nicht so benannt war, neue Konzepte entwickelt. Er hat, über die schon erwähnten Probleme mit der Tuberkulose hinaus, auch in den Überlegungen zur Pathophysiologie einen eminent wissenschaftlichen Ansatz gewählt, um die Phänome der Atmung entsprechend fassen und messen zu können. Er hat als einer der ersten an seiner Klinik Funktionsuntersuchungen durchführen lassen. Der Autor dieser Zeilen hat als Assistent am Universitätskrankenhaus Eppendorf die Bestimmung der Atemvolumina noch an einem aus Brauers Zeiten stammenden Apparat erlernt. Von Brauer stammt der Begriff der respiratorischen Insuffizienz, heute jedermann geläufig, damals als Begriff des Organversagens der Lunge, ähnlich dem von Herz und Leber, ein völlig neues Konzept [3].
Obwohl sich Brauer in Marburg sehr wohl fühlte ( $\bullet$ Abb. 1 zeigt ein Bild aus dieser Zeit) und dort auch bleibende Strukturen hinterlassen hat - der heute noch bestehende Kurhessische Verein für Luftfahrt geht auf seine Initiative zurück - hatte er doch viel weitergehende Konzepte für eine wissenschaftliche Medizin, die er nicht glaubte in der schon damals beengten Situation Oberhessens realisieren zu können. Er nahm deswegen 1910 den Ruf als Nachfolger von Hermann Lenhartz, der einer perniziösen Anämie zum Opfer fiel, als Direktor des Allgemeinen Krankenhauses Eppendorf in Hamburg an, um seine Ideen von einer forschungsbezogenen Medizin verwirklichen zu können, zumal seinerzeit die Gründung einer Universität in Hamburg geplant war. Einen gleichzeitigen Ruf nach Greifswald lehnte er ab. Die Universität wurde aber erst 1920 realisiert. Ludolph Brauer hatte sehr dezidierte Vorstellungen über die akademische Medizin und wollte eher ein „akademisches“ Krankenhaus als die Einbindung in eine Universität (s.u.). Er wollte eine akademische Medizin, die von nichtwissenschaftlichen Zwängen wie Lehre und Verwaltung befreit ist, um sich dem klinischen Fortschritt wirklich uneingeschränkt widmen zu können. Er selber war, wie Zeitzeugen berichten (unter anderen der klinische Lehrer des Autors, H. Bartelheimer), ein herausragender klinischer Lehrer, was kaum verwundert, da er als allgemeiner Internist über einen sehr breiten klinischen Horizont verfügte, sonst hätte er auch seinen Aufgaben als Direktor eines der größten Krankenhäuser Europas nicht nachkommen können. Sein Konzept sah eine Klinik vor, die im Dienste der Forschung gestanden hätte, in der Forscher freien $\mathrm{Zu}$ gang zum klinischen Material haben, ohne dass sie durch nicht forschungsrelevante Tätigkeiten belastet werden, und deren Organisation auch nur diesem Ziel dienen sollte. Es ist bemerkenswert, dass Elemente dieser Überlegungen auch heute, 100 Jahre später, in den Bekundungen der entsprechenden Wissenschaftsorganisationen insbesondere im Blick auf die USA immer wieder erkennbar sind, wobei, kritisch angemerkt, bis heute nicht geklärt ist, welche Strukturen tatsächlich am effektivsten für die Förderung von Forschung sinnvoll sind. Interessant zu erwähnen ist, dass die Flexner-Kommission, die - wie bekannt - die medizinischen Fakultäten in den USA nachhaltig reformiert hat, eine Reform, von der die US-amerikanische Medizin heute noch profi- 
tiert, zu Zeiten von Brauer 1922/23 in Eppendorf war. Für den Wechsel von Marburg nach Hamburg war wohl auch Brauers Hoffnung mitentscheidend, dass seine Vorstellungen in einer neu geschaffenen Struktur - das Allgemeine Krankenhaus Eppendorf war damals gerade erst 20 Jahre alt - besser und leichter zu realisieren wären, als in den Strukturen einer fast 400 Jahre alten Universität.

Brauers Vorstellungen bezogen sich auf eine forschungszentrierte Medizin. Er etablierte im Krankenhaus Eppendorf Forschungsbereiche, die es so noch nicht gegeben hatte. Diese Forschungsinstitute reflektierten die umfassenden wissenschaftlichen Interessen von Ludolph Brauer ebenso wie die grundlegenden wissenschaftlichen Fragen der Zeit. Naturgemäß waren aber die Institute, die sich mit Fragen der Lunge befassten, im Vordergrund des Interesses Brauers. Ludolph Brauer war genial und erfindungsreich auch in der Aufsuchung von Finanzquellen, wobei er damals sogar die gesetzliche Krankenversicherung zur Forschungsunterstützung bewegen konnte, ein Sachverhalt, der heute, kaum verständlich als Quelle von Forschungsmitteln, unendliche Diskussionen erzeugt, ohne ein weiterführendes Ergebnis zu generieren. Wesentliche Ressourcen seiner damaligen Forschung kamen wie schon erwähnt von den Landesversicherungsanstalten, aber seinerzeit gut begründet durch das Ziel der Verbesserung der Behandlung der Patienten. Innerhalb kurzer Zeit kamen diese extern finanzierten Bereiche zu großer Bedeutung. 1912 wurde das Krebs- und Tuberkuloseforschungsinstitut und 1913 ein Institut für pathologische Physiologie geschaffen. Die „Deutsche Forschungsanstalt für Tuberkulose" hatte Einrichtungen zum Studium klimatischer Heilfaktoren, pneumatische Kammern, die Über- oder Unterdruckexpositionen ermöglichten zur Untersuchung von Asthmakranken oder Patienten mit Nachwirkungen einer Gasvergiftung oder solche mit Bronchitis oder Bronchiektasen. Die Forschung betraf auch die Anwendung erhöhter Sauerstoffspannung oder den Einfluss ultravioletter Strahlung. Es wurden Langzeituntersuchungen der Ventilation durchgeführt, ein methodischer Ansatz, der erst Anfang der 80er-Jahre in der Klinik des Autors wieder aufgegriffen wurde. Hier können nur einige Beispiele genannt werden, Vollständigkeit ist nicht möglich und nicht beabsichtigt; erwähnenswert ist aber auch, dass Brauer auch der Krankenernährung Aufmerksamkeit schenkte und eine der ersten speziellen Diätküchen einrichtete, in der auch die Ärzte Kochen lernen mussten [1]. In ihrer Großzügigkeit und ihren Ergebnissen waren diese Forschungseinrichtungen auf der Welt einzigartig. 1913 gelang es durch eine großzügige Spende des Dermatologen Prof. Hugo Plaut, sein Name lebt in der Angina Plaut-Vincent fort, ein Forschungsinstitut für Mykologie zu etablieren. Die Idee von Forschungsinstituten war in jener Zeit, nicht zuletzt durch die Gründung der Kaiser-Wilhelm-Gesellschaft, der jetzigen Max-Planck-Gesellschaft, sehr populär, sodass Brauer mit seinen Konzepten durchaus Anklang fand. Er hat diese Konzepte auch mehrfach publiziert, allerdings ließ sich diese Struktur in der von ihm geforderten Stringenz in einem öffentlichen Stadtkrankenhaus, Hamburg hatte damals noch keine Universität, nicht völlig durchsetzen, was zu einer Reihe von Misshelligkeiten im Laufe der Jahre geführt hat. Damals war Hamburg ein Modellkrankenhaus für die Frage, wie man die akademische Medizin organisieren sollte. Flexner hat nicht zuletzt in Hamburg seine Ideen für eine Neuordnung der amerikanischen Fakultäten gewonnen.

Auf dem Gebiet der Inneren Medizin, speziell der Erforschung der Lungenkrankheiten, wurde die Eppendorfer Klinik zu einer weltweit anerkannten Forschungsstätte, die eine Vielzahl in- und aus- ländischer Wissenschaftler anzog, zumal in den Forschungsinstituten auch Stipendien zur Verfügung standen. Seine Arbeiten über die Pneumonie und über diffuse Lungenparenchymerkrankungen unter dem Begriff Pneumonose, der sich leider nicht durchgesetzt hat, beschreiben Sachverhalte, die erst wieder in den sechziger Jahren unter dem Begriff des ARDS Aufmerksamkeit fanden. Diese Arbeiten unterstreichen den Begriff der „Respiratorischen Insuffizienz“, die sich diesem Konzept unmittelbar anschließt [3]. Brauer unterscheidet bereits die zentrale respiratorische Insuffizienz von Formen, die sich durch Obstruktion oder Restriktion ergeben (diese Begriffe waren damals allerdings noch nicht bekannt), und solchen Formen, die sich aus Veränderungen der Luft- und Blutverteilung herleiten, mithin heute als Verteilungsstörung oder Störung des Belüftungs-Durchblutungsverhältnisses bezeichnet werden. Die späteren Arbeiten von Knipping (nachmalig Direktor der Medizinischen Klinik der Universität Köln) bauen auf diesen Ergebnissen auf. Auch der Begriff der Kurzschlussdurchblutung wird bereits geprägt. Es ist - worauf schon an anderer Stelle hingewiesen wurde - nicht möglich, alle wissenschaftlichen Leistungen hier aufzuzählen und zu würdigen. Die angeführten mögen als Beispiele für einen besonders regen, innovativen und produktiven Geist dienen, der Ludolph Brauer war.

Den Untersuchungen von Ludolph Brauer ist es zu verdanken, dass die Methode der Pneumothoraxbehandlung, die von Carlo Forlanini 1894 angegeben worden war, tatsächlich in die klinische Praxis umgesetzt werden konnte, eine Methode, die bis zur Entdeckung der Tuberkulostatika eine der ganz wenigen Möglichkeiten war, außer Operation und dem gelegentlichen Spontanverlauf, zu einer Heilung der Erkrankung zu kommen. Der Begriff Kollapstherapie stammte aus Hamburg. Brauer und seine Schüler haben in systematischen Arbeiten die Durchführbarkeit der Pneumothoraxbehandlung bewiesen und für ihre weltweite Akzeptanz die Grundlagen gelegt, auch den Einsatz radiologischer Verfahren bei der Pneumothoraxanlage und -kontrolle schon 1906 für wesentlich gehalten. Nicht zuletzt wegen dieser Arbeiten wurden ihm viele Ehrungen zuteil. Er war Ehrendoktor der Universitäten Bonn, Cordoba, Shanghai und Istanbul und Ehrenmitglied in 51 wissenschaftlichen Gesellschaften, verteilt über die ganze Welt.

Das Brauersche Forschungsideal bestand ursprünglich in einer Klinik, die das forschungsrelevante Material liefern sollte, aber befreit sein sollte von nichtwissenschaftlichen Aufgaben. Da das nicht grundsätzlich durchführbar war, sind die von Brauer etablierten Forschungsinstitute auf dem Gelände des Universitätskrankenhauses in gewisser Weise als Kompensation aufzufassen. Ludolph Brauer ist es gelungen, diese Hamburger Forschungsinstitute zu international führenden Zentren der Atmungsphysiologie und der Therapie von Lungenerkrankungen zu machen. Leider hat sein Nachfolger daran nicht anknüpfen können.

1922 war Ludolph Brauer Vorsitzender der Deutschen Gesellschaft für Innere Medizin und setzte sich mit dem damals beginnenden Sozialstaat auseinander [2]. Fast prophetisch schrieb er: „In Deutschland drängen die heutigen Verhältnisse (1922!) in dem ungesunden Kampf zwischen den Kassenbetrieben und den organisierten Ärzten zwei Gefahren entgegen: Ärztestreik oder Übernahme von medizinischen Aufgaben durch die Kassen“. Leider ist beides, und noch viel mehr, eingetreten.

Ausführlich befasste sich Brauer in seiner Präsidialrede als Vorsitzender der Deutschen Gesellschaft für Innere Medizin in Wiesbaden mit der akademischen medizinischen Ausbildung und forderte, dass der akademische Unterricht eng mit der Forschung 
verknüpft werden müsste, zur Heranbildung einer neuen Generation von akademischen Lehrern mit Charakter und Wissen. „Wir brauchen an den Universitäten nicht nur Gelehrte, sondern Persönlichkeiten“. Das ist ein Thema, das gerade jetzt sehr aktuell ist, denn gegenwärtig (2008) gehen wir genau in die andere Richtung und gestalten das Medizinstudium in Richtung eines Fachschulstudiums. Brauer 1922: „Die Ziele werden nicht erreicht durch neue Unterrichtspläne und kleine Reformen und die Einbeziehung aller möglichen Nachbarfächer oder Gruppenvertreter ... Je mehr in unser akademisches Leben wirtschaftliche Rücksichten hineingetragen werden, desto mehr wird es nötig sein, rein wissenschaftliche Arbeitsstätten möglichst frei auszugestalten“. Leider ist die Medizin in Deutschland, und auch die akademische Medizin, in den letzten Jahren in die gegensätzliche Richtung gezwungen worden.

Ludolph Brauer, obwohl als Direktor des Universitätskrankenhauses Eppendorf auf Lebenszeit bestimmt, wurde 1934 unter unwürdigen Bedingungen entlassen. Er verlegte die Deutsche Forschungsanstalt für Tuberkulose von Hamburg nach Wiesbaden und baute später in Frankfurt eine Forschungsanstalt für Aufbrauch- und Alterskrankheiten auf, die 1944 dem Bombenterror zum Opfer fiel. Mit den Resten dieses Projekts zog er sich nach Bayern zurück, wo er in einfachen Verhältnissen an einer zunehmenden Herzinsuffizienz am 25.11.1951 verstarb.
Was hinterlässt uns Ludolph Brauer? Den Glauben an die Bedeutung einer originären und originellen Wissenschaft für die Behandlung unserer Patienten, die Überzeugung, dass Grundlagenwissenschaften für dieses Ziel eine hohe Bedeutung haben, und die Überzeugung, die klinische Forschung an die klinische Medizin und deren Probleme zu knüpfen. Für die wissenschaftliche Pneumologie, einen Begriff, den Ludolph Brauer noch nicht kannte, weil er Internist war, bedeutet das, dass er gezeigt hat, dass die Wissenschaft von den Erkrankungen der Lunge im absoluten Zentrum der Inneren Medizin steht, aber nur dort auch langfristig überleben kann.

\section{Literatur}

1 Hein J. Ludolph Brauer - Der Wegbereiter der modernen Pneumologie. Prax Klin Pneumol 1984; 38: 471 - 473

2 Brauer L. Eröffnungsrede zur 34. Tagung der Deutschen Gesellschaft für Innere Medizin. In: H. G. Lasch, B. Schlegel (Hrsg). Hundert Jahre Deutsche Gesellschaft für Innere Medizin. München: J. F. Bergmann, 1982

3 Brauer L. Die respiratorische Insuffizienz. In: Verh. Der Deutschen Gesellschaft für Innere Medizin, 44. Tagung. München: J. F. Bergmann, 1932

4 Brauer L. Die praktische Durchführung des Überdruckverfahrens. Dtsch Med Wschr 1905; 31: 187-192

5 Brauer L. Antrittsvorlesung als Direktor der Medizinischen Klinik der Philipps-Universität Marburg. Dtsch Med Wschr 1905; 31: 1801 1805 